\title{
Effects of Experimental Parameters on the Growth of GaN Nanowires on Ti- film/Si(100) and Ti-foil by Molecular Beam Epitaxy
}

K. Mudiyanselage, K. Katsiev, and H. Idriss*

Surface Science and Advanced Characterization, SABIC-Corporate Research and Development (CRD) Center at KAUST, 23955-Thuwal, Saudi Arabia

\begin{abstract}
Gallium nitride $(\mathrm{GaN})$ nanostructures are used in optoelectronic applications due to their unique optical and electronic properties. For some optoelectronic applications and potential photocatalytic systems, the growth of GaN nanowires on metallic substrates instead of expensive single crystalline semiconductors can be beneficial due to specific properties of metals. In this study, GaN nanowire systems were grown on $300 \mathrm{~nm}$ Ti-film/Si(100) and Ti-foil by plasma assisted molecular beam epitaxy (PA-MBE) and characterized in situ by Auger electron spectroscopy (AES) and ex situ by scanning electron microscopy (SEM). Effects of (i) the nature of substrate surface, (ii) Ga flux, and (iii) substrate temperature on the growth of GaN nanowires were investigated. Nearly vertical nanowires can be grown on Ti-films covered with amorphous $\mathrm{TiO}_{x}$ or $\mathrm{TiO}_{x} \mathrm{~N}_{y}$, which is formed during the nitridation process. To grow nearly vertical nanowires on Ti-foils, pre-nitridation of the substrate surface was found to be important. The orientation of GaN nanowires grown on nitridated Ti-foil is determined by the grain alignment of the original Ti-foil, however, GaN nanowires grown on nitridated Ti-foils are uniformly oriented to one direction within an individual grain, which is most likely due to the epitaxial relation between the nanowires and the underneath grains of the polycrystalline Ti-foils. Both the orientation and nanowire density vary on different grains.
\end{abstract}

\section{Key words:}

GaN nanowires; Ti foil nitridation; in situ AES of film growth; Ti oxynitride layers; nanowires orientation.

*Corresponding author's Email: idrissh@sabic.com 


\section{Introduction}

In general, both thin films and nanostructure materials are primarily grown on single crystalline substrates. Gallium nitride $(\mathrm{GaN})$ nanowires are mainly grown on single crystalline Si substrates [1-10]. The use of metallic substrates can be beneficial for some optoelectronic applications due to specific properties of metals such as efficient thermal conductivity, and charge carrier extraction. A few recent studies reported the growth of GaN nanowires on Ti-films [11, 12] and foils [13-18]. In most of these studies, a TiN layer was prepared before growing GaN nanowires [11-14, 16-18]. While a TiN layer is not intentionally prepared before growing nanowires, it may form during the growth of $\mathrm{GaN}$ from the reaction between impinging $\mathrm{N}$ and $\mathrm{Ti}$ on the substrate. TiN is stable, conductive [12], and it makes an ohmic contact with GaN[13, 19, 20].

Depending on experimental conditions and substrate surface properties, GaN nanowires grow in different orientations with respect to the substrate surface normal. For some applications, vertically oriented nanowires are desirable but nanowires grown on metallic substrates generally have a broad distribution of tilt angles with respect to the substrate normal [15]. The mechanism and reaction conditions responsible for the growth of vertical nanowires is not completely clear. A few studies investigated the mechanism that control the orientation of $\mathrm{GaN}$ nanowires grown on Ti metal foils [14, 15]. It has been reported that $\mathrm{GaN}$ nanowires grown on metal foils are uniformly tilted with respect to the surface normal within individual grains of polycrystalline metal foils[15]. The distribution of tilt angles of nanowires grown on Ti-foil has been attributed to an epitaxial relation between the nanowires and the individual grains of the polycrystalline metal foil[15]. However, the details of such a relationship are not yet completely understood.

The effects of surface roughness, chemical composition, and native oxide crystallinity on the orientation of $\mathrm{GaN}$ nanowires grown on Ti-foils have been reported previously[14]. Calabrese et al. reported that regardless of the substrate crystallinity, an initially smooth surface is not sufficient to guarantee the vertical growth of GaN nanowires on Ti-foils. The observed random orientation of nanowires on Ti- foils was attributed to increased surface roughness of the foils due to surface reactions between the impinging elements ( $\mathrm{Ga}$ and $\mathrm{N}$ ) with Ti[14]. Therefore, the protection of the substrate surface from undesired chemical reactions, which lead to the surface roughness, by passivation with pretreatment was required to obtain vertically grown nanowires[14]. Since a TiN layer can act as a passivation layer for the Ti surface against chemical reactions, a TiN layer was grown prior to the growth of $\mathrm{GaN}$ nanowires in previous studies $[14,15]$. The growth of TiN is a straightforward approach since 
an $\mathrm{N}$ source, which is used for the growth of $\mathrm{GaN}$ nanowires, is already available. Therefore, a TiN layer, which is stable, conductive, and can form an ohmic contact with $\mathrm{GaN}[12,19,21]$, can be grown easily by exposing the Ti-foil to $\mathrm{N}$ plasma.

Calabrese et al. reported that on nitridated Ti-foils, uniformly tilted nanowires were grown on the surface of each individual grain of the polycrystalline substrate [14]. These results are consistent with the findings reported by May et al., where they also observed uniformly tilted GaN nanowires at the surface of individual grains of Ti-foils[15]. These preferred growth directions vary across the sample, which was attributed to the polycrystalline nature of the foil with grains in the range of tens to a few hundred micrometers. Based on the observed results, Calabrese et al. concluded that the orientation of GaN nanowires grown on nitridated Ti metal substrate is not determined by the surface roughness, but by the crystallinity of the original foil[14].

When a native $\mathrm{TiO}_{x}$ layer is present on Ti-foils, the orientation of nanowires depends on the nature of the oxide layer. It was reported that when the native oxide layer is crystalline, GaN nanowires grow homogeneously tilted at the surface of each individual grain composing the substrate[14]. In contrast, GaN nanowires grow vertically when the foil is covered by a thin amorphous oxide layer, which was ascribed to an interruption of the epitaxial information from the underlying substrate[14]. Therefore, the presence of an amorphous surface oxide layer prevents epitaxial growth hence nanowires grow vertically all across the substrate [14]. This result is also consistent with that reported in previous studies on oxide layers, where vertically orientated $\mathrm{GaN}$ nanowires were grown on amorphous $\mathrm{Si}_{\mathrm{x}} \mathrm{O}_{\mathrm{y}}$ and $\mathrm{Al}_{\mathrm{x}} \mathrm{O}_{\mathrm{y}}$ layers deposited on crystalline Si substrates[6, 22].

When $\mathrm{GaN}$ nanowires are grown on amorphous $\mathrm{TiO}_{2}$, which has a high electrical resistivity, the effective charge carrier injection from the Ti-foil into $\mathrm{GaN}$ nanowires would take place in the presence of defects or through electron tunneling, which strongly depends on the thickness of the oxide layer[23]. One of the alternative ways to obtain vertically oriented nanowires on a Ti-foil or Ti-film with a native oxide layer, without sacrificing the benefit of conductivity, would be the growing of an amorphous conducting interlayer, such as a $\mathrm{TiO}_{\mathrm{x}} \mathrm{N}_{\mathrm{y}}$ [24]. When a native amorphous oxide layer is present on $\mathrm{Ti}$ substrates, the nitridation leads to form $\mathrm{TiO}_{x} \mathrm{~N}_{y}$, which has been proposed to be a suitable interlayer [14].

In general, nanowire systems prepared on Ti-foils are relatively less dense, and the diffusioninduced repulsion of neighboring nanowires was proposed as the main mechanism limiting nanowire density during nucleation on TiN[11]. In diffusion-induced repulsion, once a nanowire nucleates, adatoms diffuse towards the nucleus hence the adatom concentration in the surrounding area decreases. 
Therefore, the probability for further nucleation close to the already formed nanowire is reduced. The adatom diffusion length on the substrate determines the density of nanowire systems[11].

In this study, GaN nanowires were grown with and without pre-nitridation of $300 \mathrm{~nm}$ Ti-films/ n-Si(100) as well as Ti-foils to identify the impact of substrate nitridation on the orientation of GaN nanowires. On Ti-films, GaN nanowires were grown without removing the native oxide layer to see its effect on the growth of nanowires. The native oxide layer on Ti-foil was removed by Ar ion sputtering and GaN nanowires were grown on cleaned Ti-foils with and without pre-nitridation. The effects of $\mathrm{Ga}$ flux, and substrate temperature on the growth of GaN nanowires were also investigated. In addition, nanowires were also grown on both polished and unpolished Ti-foils.

\section{Experimental}

GaN nanowires were grown in a built on purpose plasma-assisted molecular beam epitaxy (PAMBE) system with an effusion cell (CreaTec Fischer \& Co.) for gallium (Ga) and a RF-plasma source (PCS-RF, SPECS) for $\mathrm{N}$ atoms. The base pressure of the PA-MBE system is $\sim 3.0 \times 10^{-10}$ Torr and it is also equipped with Auger electron spectroscopy (AES - STAIB, ESA 100), mass spectrometry (SRS, RGA 200), and Ar ion sputtering (Ion Source IQE 11/35, SPECS). Ga pellets (purity: 99.9999\%, Alfa Aesar) was used in the effusion cell. During the growth of GaN nanowires, the nitrogen pressure in the chamber was kept $\sim 3.0 \times 10^{-5}$ Torr and the power of the RF-plasma source was set at $350 \mathrm{~W}$. All the $\mathrm{GaN}$ nanowire systems were grown under nitrogen rich conditions. GaN nanowires were grown on 300 nm Ti-film/n-Si (100) and Ti-foils (10x10x0.5 mm) [unpolished Ti-foil, purity $\approx 99 \%$ (Alfa Aesar) and one side polished Ti-foil, purity> 99.9\%, (MTI Corporation)]. The $300 \mathrm{~nm}$ Ti-film on n-Si(100) was deposited at $25{ }^{\circ} \mathrm{C}$ by e-beam evaporation of $\mathrm{Ti}$ in a separate vacuum chamber. The temperature of the home built sample stage was measured by a type-K thermocouple attached to it whereas the substrate temperature was measured by a pyrometer. The temperature of the $300 \mathrm{~nm}$ Ti-film/n-Si(100) substrate was found to be $\approx 150{ }^{\circ} \mathrm{C}$ higher than the thermocouple temperature $\left(\mathrm{T}_{\mathrm{tc}}\right)$ whereas the temperature of the Ti-foil substrate was $\approx 200{ }^{\circ} \mathrm{C}$ higher than the $\mathrm{T}_{\mathrm{tc}}$ as determined by measuring the substrate temperature with a pyrometer. Substrate temperatures measured with the pyrometer, $\mathrm{T}_{\text {sub }}$, are reported here. The 300 $\mathrm{nm}$ Ti-film/n-Si(100) substrate was cleaned by annealing at $\mathrm{T}_{\text {sub }}=350{ }^{\circ} \mathrm{C}$ for $\approx 30$ minutes whereas the Ti-foil substrate was cleaned by Ar ion sputtering for $\approx 2$ hours to remove carbon, sulfur, and calcium contaminants as well as a native oxide layer and subsequent annealing at $\mathrm{T}_{\mathrm{sub}}=650{ }^{\circ} \mathrm{C}$ for $\approx 30 \mathrm{~min}$. GaN nanowire systems were characterized in situ by Auger electron spectroscopy (AES) and ex situ by 
scanning electron microscopy (SEM). Quantitative determination of the different elements by AES was conducted using the peak-to-peak height after correction to the relative sensitivity factors (see Fig. 1).

\section{Results and Discussion}

\subsection{GaN nanowires on Ti-film}

\subsubsection{Growth of GaN nanowires on $300 \mathrm{~nm} \mathrm{Ti-film/n-Si(100)}$ at $680{ }^{\circ} \mathrm{C}$}

Figs. 1A and 1B present plan and $40^{\circ}$-tilt views of SEM micrographs of GaN nanowires grown

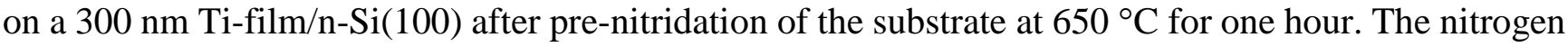
plasma source operating condition during nitridation is the same as that used for the growth of GaN nanowires. The GaN nanowire system shown in Fig. 1 was grown at a substrate temperature, $\mathrm{T}_{\text {sub, }}$ of 680 ${ }^{\circ} \mathrm{C}$ and a Ga effusion cell temperature, $\mathrm{T}_{\mathrm{Ga}}$ cell, of $865^{\circ} \mathrm{C}$. Relatively dense nanowires are seen with lengths in the range of $\approx 280-320 \mathrm{~nm}$. Growth parameters and properties of GaN nanowire systems on Ti-film/n-Si(100) are shown in Table 1. A large fraction of the substrate surface is covered by uniformly distributed nanowires with some coalescence. The heterogeneity of the density is most likely due to the changes in the morphology of the Ti-film underneath. Fig. 1C presents the Auger electron spectrum for the GaN nanowire system shown in Fig. 1A. In addition to the $\mathrm{N}$ (KLL), Ti(LMM) and Ga(LMM) a less intense peak for $\mathrm{O}(\mathrm{KLL})$ is seen, originating from a native Ti oxide layer on the Ti-film before the growth of $\mathrm{GaN}$. The oxide layer was formed on the Ti-film when it was exposed to air during the transfer from the Ti-film preparation chamber to the PA-MBE system. The peak-to-peak height ratio of $\mathrm{N}(\mathrm{KLL}) / \mathrm{Ga}(\mathrm{LMM})$ after incorporating the Auger sensitivity factors [Ga(LMM) - 0.14 and N(KLL) $0.35]$ is $\approx 1.0(\mathrm{Ga}: \mathrm{N} \approx 1.0: 1.0)$. The peak-to-peak height ratio of $\mathrm{O}(\mathrm{KLL}) / \mathrm{Ga}(\mathrm{LMM})$ after incorporating Auger sensitivity factors $[\mathrm{O}(\mathrm{KLL})-0.50]$ is $0.03(\mathrm{Ga}: \mathrm{O} \approx 1.00: 0.03)$.

\subsubsection{Effect of nitridation}

To study the effect of nitridation, two GaN nanowire systems were prepared at exactly the same conditions with and without pre-nitridation and their SEM micrographs are presented in Figs. 2A and 2C. Fig. 2A presents the SEM micrograph of GaN nanowires grown after pre-nitridation with the Ga cell temperature of $860^{\circ} \mathrm{C}$. The AES Ga: $\mathrm{N}$ is found to be $1.0: 1.4$ while the Ga:O was $1.0: 0.1$. Lengths of nanowires are in the range of $\approx 150-210 \mathrm{~nm}$ whereas their diameters are in the range of $\approx 50-70 \mathrm{~nm}$. While the nanowires covered a large fraction of the substrate surface, a significant fraction of the surface was empty. SEM micrograph and Auger electron spectrum for the GaN system prepared without pre- 
nitridation are shown in Figs. 2C and 2D, respectively. The SEM micrograph in Fig. 2C shows that a large fraction of the substrate surface is covered with a dense nanowire system. Lengths of nanowires are in the range of $\approx 230-270 \mathrm{~nm}$ whereas their diameters are in the range of $\approx 60-100 \mathrm{~nm}$. The AES Ga:N ratio in this system is $\approx 1.0: 1.0$ (which indicates that no excess $\mathrm{N}$ originating from $\mathrm{TiN}$ is seen, most likely because of the high density of the nanowires). No AES O(KLL) signal was detected.

The results shown in Figs. 1 and 2 indicate that nearly vertical nanowires are grown on Ti-film with or without pre-nitridation. An oxide layer is present on the Ti-film before the growth of GaN nanowires (AES). Other groups have reported that nanowires are grown vertically on amorphous oxide layers [14], by analogy we deduce that the oxide layer present on $300 \mathrm{~nm}$-Ti film might be amorphous. During the growth of $\mathrm{GaN}$ nanowires, $\mathrm{TiO}_{\mathrm{x}} \mathrm{N}_{\mathrm{y}}$ may also be formed. Nanowire density is lower, their lengths are shorter, and their diameters are narrower when prepared after pre-nitridation compared to without nitridation. In other words, denser, longer, and wider nanowires are formed on amorphous $\mathrm{TiO}_{\mathrm{x}}$ when compared to those grown on $\mathrm{TiO}_{\mathrm{x}} \mathrm{N}_{\mathrm{y}}$. These results suggest that the diffusion length of $\mathrm{Ga}$ adatoms, which determines the nanowire number density[11], is shorter and its desorption rate is lower on $\mathrm{TiO}_{\mathrm{x}}$ compared to $\mathrm{TiO}_{x} \mathrm{~N}_{y}$. The formation of a less dense nanowire system on $\mathrm{TiO}_{x} \mathrm{~N}_{y}$ may also be attributed to the presence of more diffusion-induced repulsion. In diffusion-induced repulsion, once a nanowire nucleates, surrounding adatoms diffuse towards the nucleus of the nanowire reducing the adatom concentration and the probability of nucleation in close proximity to the already formed nanowire.

\subsubsection{Effect of Ga flux}

To study the effect of Ga flux on the growth of nanowires, three GaN systems were prepared at the same substrate temperature, $\mathrm{T}_{\mathrm{sub}}=680^{\circ} \mathrm{C}$, with different $\mathrm{Ga}$ effusion cell temperatures at 850,840 and $830^{\circ} \mathrm{C}$. Fig. S1 in supplementary information presents the SEM micrographs of the GaN nanowires. As the Ga effusion cell temperature decreases, the lengths, and the density of nanowires decrease.

\subsubsection{Effect of substrate temperature}

Fig. 3 presents SEM micrographs of plan and $40^{\circ}$-tilt views of $\mathrm{GaN}$ nanowire systems grown with Ga cell temperature of $860^{\circ} \mathrm{C}$ and a higher substrate temperature, $\mathrm{T}_{\text {sub }}=700^{\circ} \mathrm{C}$. Nanowires with slightly lower density than that in Fig. 2C, where the substrate temperature is lower by $20^{\circ} \mathrm{C}$, is seen. Nanowires in this system are aligned vertically. The nanowire lengths are in the range of $200-240 \mathrm{~nm}$, which is similar to those in the system in Fig. 2C and their diameters (in the range of 40-70 nm) are smaller. These results show that thinner nanowires are grown at higher substrate temperature. In general, higher 
substrate temperatures induce $\mathrm{Ga}$ adatoms desorption and diffusion hence relatively thin and less dense nanowires are grown. However, still the nanowire density is high; Ti was not detected by AES (Fig. 3C).

\subsection{GaN nanowires on Ti-foil}

\subsubsection{Growth of GaN nanowires on Ti-foil at $650^{\circ} \mathrm{C}$}

Prior to the growth of $\mathrm{GaN}$, the Ti-foil substrate was cleaned as described in the experimental section. Fig. 4 presents SEM micrographs of plan and $40^{\circ}$-tilt views of $\mathrm{GaN}$ nanowires grown without pre-nitridation at $700{ }^{\circ} \mathrm{C}$. Growth parameters and properties of $\mathrm{GaN}$ nanowire systems on Ti-foils are shown in Table 2. Less dense and randomly oriented nanowires are seen. These results agree with a previously reported study where the observed random orientation of nanowires on Ti-foils was ascribed to the increased surface roughness of the foils due to the reactions between impinging $\mathrm{Ga}$ and $\mathrm{N}$ with Tifoil[14]. AES O(KLL) signal was not seen (Fig. 4C). During the growth of GaN nanowires, a TiN is also formed by the reaction of Ti with impinging N. Since the nanowire density is low, the N in TiN was detected by AES; the Ga:N ratio is 1.0 : 2.2 .

\subsubsection{Effect of nitridation}

Similar growth as the previous section was conducted after pre-nitridation at $700{ }^{\circ} \mathrm{C}$ for one hour, and its SEM micrographs of plan and $40^{\circ}$-tilt views are shown in Fig. 5. Vertical but less dense nanowires are seen. These results are in line with previous studies $[11,14]$, which show that a less dense and vertically aligned nanowires grow on TiN. The AES N (KLL), Ti(LMM) and Ga(LMM) lines are presented in Fig. 5C, no lines related to carbon or oxygen atoms were present. Results presented in Figs. 4 and 5 indicate that the pre-nitridation is needed to obtain relatively dense and nearly vertical nanowires on Ti-foils.

\subsubsection{Effect of substrate temperature and Ga flux}

To see the effect of Ga flux and substrate temperatures, GaN nanowire systems were grown after the pre-nitridation. Fig. 6A presents the SEM micrograph of the $\mathrm{GaN}$ nanowires prepared at $\mathrm{T}_{\text {sub }}=680$ ${ }^{\circ} \mathrm{C}$ with the Ga cell temperature of $835^{\circ} \mathrm{C}$. AES of this system together with that of Fig. 5A are shown in Fig. 6B. The amount of Ga detected by AES in both systems is similar but lengths of nanowires are longer with the growth at higher substrate temperature and higher $\mathrm{Ga}$ flux $\left(\mathrm{T}_{\mathrm{sub}}=700{ }^{\circ} \mathrm{C}, \mathrm{T}_{\mathrm{Ga}}\right.$ cell $=865{ }^{\circ} \mathrm{C}$, length $\approx 220-305 \mathrm{~nm} ; \mathrm{T}_{\text {sub }}=680{ }^{\circ} \mathrm{C}, \mathrm{T}_{\mathrm{Ga}}$ cell $=835^{\circ} \mathrm{C}$, length $\left.\approx 120-200 \mathrm{~nm}\right)$. However, nanowire 
diameters are smaller at $\mathrm{T}_{\text {sub }}=700{ }^{\circ} \mathrm{C}(\sim 30-60 \mathrm{~nm})$ than those of nanowires grown at $\mathrm{T}_{\text {sub }}=680{ }^{\circ} \mathrm{C}(\sim$ 60-100 nm). These results show that both Ga flux and substrate temperature under a constant $\mathrm{N}$ flux affect the lengths and diameters of nanowires hence these two parameters need to be optimized to obtain nanowire systems with desired properties.

To see the effect of higher Ga flux (higher Ga effusion cell temperature), the Ga cell temperature of $875^{\circ} \mathrm{C}$ was used; SEM micrographs of plan and $40^{\circ}$-tilt views are presented in Fig. 7. Dense and inhomogeneous nanowires are formed. In addition, less hexagonal shaped nanowires are present when compared with those shown in Fig. 5. Hexagonal shaped nanowires with higher crystallinity are therefore preferentially grown in nitrogen rich conditions.

GaN nanowires were also grown on polished Ti-foil with and without pre-nitridation. SEM micrographs of a $\mathrm{GaN}$ nanowire system prepared on a polished Ti-foil without pre-nitridation at $700{ }^{\circ} \mathrm{C}$ are shown in Fig. 8. The majority of nanowires are randomly oriented away from the surface normal but some nanowires are aligned vertically as shown by Figs. 8A and 8B. These results indicate that the initial surface smoothness of the substrate is not sufficient for the growth of vertically aligned GaN nanowires on Ti-foils as reported previously[14]. The nanowire density is much lower too. The large area SEM micrograph in Fig. 8C shows that the nanowire density varies significantly on different grains.

SEM micrographs of a GaN nanowire system grown after nitridation are shown in Fig. 9. After forming a TiN layer on the Ti-foil with pre-nitridation, less dense and hexagonal shaped nanowires are grown. The nanowires are uniformly tilted with respect to the surface normal and oriented along one direction. The growth of nanowires that were uniformly tilted with respect to the surface normal within the individual grains on metal foils was previously attributed to an epitaxial relation between the nanowires and the individual grains of polycrystalline metal foils[15]. It was also reported that these preferred growth directions vary across the sample, which was attributed to the polycrystalline nature of the foil with grains of tens to a few hundred micrometers. Previous studies have reported that GaN nanowires grow perpendicular to $\mathrm{TiN}(111)$ with the epitaxial relation, $\mathrm{GaN}(0001)<11$ $20\rangle \| \operatorname{TiN}(111)\langle 110\rangle[12,25,26]$. Most of localized defects are present at grain boundaries, separating them with different alignments. Because the $\{111\}$ facets of TiN grains of the nitridated Ti-foil are not parallel, the nanowires grown perpendicular to $\operatorname{TiN}(111)$ with this epitaxial relation, will tilt differently on each grain. Therefore, nanowire directions depend on the alignment of grains due to the epitaxial relation of nanowires and underneath $\mathrm{TiN}(111)$ surface. The $\mathrm{TiN}$ layer grown at $\approx 700{ }^{\circ} \mathrm{C}$ is crystalline[11] and affected by the properties of the underneath Ti-foil grains. Consequently, the orientation of $\mathrm{GaN}$ nanowires are determined by the grains of polycrystalline Ti-foil. The density of 
nanowires also varies on different grains of the polycrystalline Ti-foil as shown by the SEM micrograph in Fig. 9B. The diameters of the majority of nanowires in Fig. 9A are $\approx 50 \mathrm{~nm}$ whereas the lengths are in the range of $\approx 170-220 \mathrm{~nm}$.

Increasing the temperature of the Ga effusion cell to $865^{\circ} \mathrm{C}$, Fig. 10 , resulted in the formation of relatively denser nanowires, which were inhomogeneous in both lengths and diameters. The shape of the majority of nanowires are not as hexagonal as that of nanowires shown in Fig. 9A, grown at lower Ga effusion cell temperature. Therefore, N-rich growth condition is required for growing high crystalline hexagonal shaped GaN nanowires. Some nanowires coalesced to form large diameter ones. In addition, the large-scale SEM micrograph in Fig. 10C shows that the density of nanowires varies on different grains of the Ti-foil.

The nanowire density directly relates to the nucleation process, which depends on the nature of substrate surface, Ga flux and substrate temperature. Ga adatom diffusion and desorption depend on the substrate temperature. Because high temperatures increase the diffusion and desorption rates low nanowire density is obtained. Since nanowire density of $\mathrm{GaN}$ is found to be lower on $\mathrm{TiO}_{\mathrm{x}} \mathrm{N}_{\mathrm{y}}$ than on $\mathrm{TiO}_{\mathrm{x}}$, the diffusion and desorption rates of $\mathrm{Ga}$ adatoms are most likely higher on the former when compared to the latter under similar experimental parameters. In the case of bare Ti-foil, a large portion of nitrogen reacts with Ti to form Ti-N due to a preferred reaction between $\mathrm{Ti}$ and $\mathrm{N}$ rather than that of $\mathrm{Ga}$ and $\mathrm{N}$. This might be one of the causes for sparse growth of nanowires on bare Ti-foil. The sparse GaN nanowires may also be due to limited nucleation sites; specific location such as grain boundaries and defect sites. Therefore, pre-nitridation would enhance the GaN nucleation sites (less loss of $\mathrm{N}$ atoms to make TiN) resulting in high-density. Hence, the nitridation of Ti-foil seems to be a prerequisite.

At high Ga flux, nuclear density increases which also leads to dense nanowire systems. However, an increase of the Ga flux may lead to Ga accumulation at the nanowire topmost region resulting in an enhancement of the lateral growth rate increasing the nanowire top diameter[27]. In general, the nanowire density decreases by raising the growth temperature since the adatom diffusion on the substrate surface is enhanced. Similarly, the nanowire density increases by increasing Ga flux since the probability to nucleate an island with critical size is increased[28]. Comparison of systems in Figs. 7 and 9 shows that a dense nanowire system is formed with higher Ga flux at the same substrate temperature (Table 2).

The $\mathrm{Ga}$ adatom diffusion on the nanowire vertical sidewalls is also enhanced by raising growth temperature, which favors the axial growth at the expense of radial growth. Also, less coalescence takes place by raising growth temperatures as the density of nuclei is reduced according to standard island nucleation theory[3]: the density of GaN nanowires is directly related to the density of spherical cap- 
shaped islands formed at the onset of the nucleation phase. In addition, while the nucleation mechanisms leading to the formation of nanowire nuclei are driven by thermodynamics, the growth mechanisms leading to the nanowire elongation are controlled by kinetics. According to Ristic et al. [27] during the nucleation process, the substrate surface is covered by GaN islands with different sizes that leads to differences in nanowire diameter. Some nanowires can start growing earlier than others resulting in heights differences since the nucleation process does not occur simultaneously all over the substrate.

GaN nanowires growth is driven by the nucleation process due to a strong lattice mismatch between the substrate and nanowires, which takes place by the Volmer-Weber (island) growth mechanism[27]. In this process, small islands of critical radii are formed. Smaller size nuclei decay either by desorption or diffusion to other nuclei. The diameter of the nuclei increases with the substrate temperature only if the Ga flux is high enough to compensate for increased desorption rates. The nanowire systems in Figs. 2C and $3 \mathrm{~A}$ show that increasing the temperature by $20^{\circ} \mathrm{C}\left(700^{\circ} \mathrm{C}\right.$ vs $\left.680{ }^{\circ} \mathrm{C}\right)$ decreased nanowire diameters by $30-40 \%$ (Table $1,700{ }^{\circ} \mathrm{C}-40-70 \mathrm{~nm}$ and $680{ }^{\circ} \mathrm{C}-70-100 \mathrm{~nm}$ ). In addition, nanowire lengths are also shorter. This may indicate that Ga diffusion and desorption on $\mathrm{TiN}$ is faster than that on the axial diffusion along the GaN wires. This is also in line with the results of Figs. 5 and 6 where longer nanowires (Table 2; 220 - $305 \mathrm{~nm}$ vs. 120-200 nm) are formed at high temperature, but when the Ga flux is increased.

Ristic et al. [27] reported that saturation of the number of nucleation sites occurs when the average distance between nuclei equals twice the Ga adatom mean diffusion length, which depends on the growth temperature, the substrate and the $\mathrm{Ga} / \mathrm{N}$ ratio. In Fig. 9A, the average separation between most nanowires is $\approx 60 \mathrm{~nm}$. Therefore, the Ga adatom mean surface diffusion length on nitridated Ti-foil under the growth conditions of the system in Fig. 9A is ca.30 nm. For comparison, the Ga adatom diffusion length on the sidewall of GaN nanowires is ca. 40-50 $\mathrm{nm}$ [3].

In the $\mathrm{GaN}$ nanowire growth process, first, initial nucleation takes place to form $3 \mathrm{D}$ islands then the shape transforms to a nanowire like morphology. Next, both axial (along c-direction) and lateral growth takes place and $\mathrm{Ga}$ adatoms take longer time to reach the top of the nanowires with elongation, which results a decrease of $\mathrm{Ga}$ adatom density at the nanowires top. In addition, increase of nanowire diameters also leads to reducing the adatom density at the top surface. When the Ga adatom density reduces below the threshold for 2D growth (lateral growth), only nanowire elongation takes place without increasing the nanowire diameter [4]. In the system shown in Fig. 9A, the nanowire diameters at the top surface are increased by ca. $30 \%$ when compared to the bottom one (the place where elongation 
of nanowires starts) for ca. $100 \mathrm{~nm}$ long. When Ga adatom density exceeds the threshold value for 2D growth at the top facet, lateral growth takes place; increase of the nanowire diameter. However, when the axial growth is faster, the Ga adatom density does not exceed the threshold value, hence no lateral growth. Both $\mathrm{Ga}$ adatom diffusion rate along nanowire walls and $\mathrm{Ga}$ impinging from gas phase to the top surface of nanowires determine the density of $\mathrm{Ga}$ adatoms at the top. The increase of nanowire diameters in Fig. 9A indicates that the Ga adatom density exceeds the threshold value for 2D growth at the top facet and lateral growth takes place. If the adatom diffusion along the nanowire wall is high and the vertical growth rate is fast enough to keep the Ga adatom density below the threshold value, the nanowires should preserve their diameters.

It was reported that in plasma-assisted molecular beam epitaxy, GaN nanowires grown under $\mathrm{N}$ rich conditions $(\mathrm{Ga} / \mathrm{N}<1)$ at high temperatures $\left(>700{ }^{\circ} \mathrm{C}\right)$ crystallize in the wurtzite structure with the polar $\langle 0001\rangle$ axis parallel to the growth direction [4]. Therefore, nanowires grown on both Ti-films and Ti-foils are crystalline but their degree of crystallinity can be different. A single nanowire should tend to minimize its surface, and its cross-sectional shape would have perfect regular hexagons [29]. However, irregular hexagonal-shaped nanowires are also formed without coalescence. In a previous study, inhomogeneous strain was observed on GaN nanowires likely due to coalescence [29]. Coalescence may introduce structural defects in addition and the root cause of these defects is crystallographic miss-orientation between the nanowires [30].

\section{Conclusions}

Nearly vertical GaN nanowires are grown on Ti-film covered with amorphous $\mathrm{TiO}_{\mathrm{x}}$ or $\mathrm{TiO}_{\mathrm{x}} \mathrm{N}_{\mathrm{y}}$. Even though there is no significant difference on the growth of GaN nanowires on Ti-films with and without pre-nitridation, the density of nanowire systems grown on nitridated Ti-film is less than that on the as prepared film most likely due to a longer diffusion length and higher desorption rate of Ga adatoms on $\mathrm{TiO}_{x} \mathrm{~N}_{y}$ when compared to $\mathrm{TiO}_{x}$. Because no oxide layer is present on the surface of Ti-foil after cleaning, randomly oriented $\mathrm{GaN}$ nanowires are grown due to the surface roughening by reactions between Ti and impinging N. Therefore, to grow nearly vertical nanowires on Ti-foils, the formation of TiN layer by pre-nitridation is required. GaN nanowires grown on nitridated Ti-foils are uniformly oriented to one direction within an individual grain. The orientation of nanowires on each grain depends on the alignment of TiN grains of nitridated Ti-foil due to the epitaxial relation between nanowires and underneath $\mathrm{TiN}(111)$ surface. In general, the nanowire density decreases by raising the growth temperature since the adatom diffusion on the substrate surface and the desorption are enhanced reducing 
a number of nucleation sites. Similarly, the nanowire density increases by increasing Ga flux since the probability to nucleate an island with critical size is increased. However, under very high Ga flux, inhomogeneous and non-hexagonal shaped nanowires are grown. Due to a low degree of coalescence, nanowires grown on Ti-foil can have less defects and high degree of crystallinity compared with those grown on Ti-film. Nearly vertical and relatively dense nanowire systems can be grown on both Ti-foil and Ti-film with surface pretreatment and optimized experimental parameters such as Ga flux, $\mathrm{N}$ flux, and substrate temperature. 


\section{References}

[1] B. AlOtaibi, M. Harati, S. Fan, S. Zhao, H.P.T. Nguyen, M.G. Kibria, Z. Mi, Nanotechnology, 24 (2013) 175401.

[2] K.A. Bertness, A. Roshko, L.M. Mansfield, T.E. Harvey, N.A. Sanford, J. Cryst. Growth, 310 (2008) 3154-3158.

[3] V. Consonni, Phys. Status Solidi-Rapid Res. Lett., 7 (2013) 699-712.

[4] S. Fernandez-Garrido, V.M. Kaganer, K.K. Sabelfeld, T. Gotschke, J. Grandal, E. Calleja, L. Geelhaar, O. Brandt, Nano Lett., 13 (2013) 3274-3280.

[5] G. Li, Y.Y. Yao, M. Dagenais, J. Cryst. Growth, 524 (2019) 125181.

[6] T. Stoica, E. Sutter, R.J. Meijers, R.K. Debnath, R. Calarco, H. Lüth, D. Grützmacher, Small, 4 (2008) 751-754.

[7] P. Varadhan, H.C. Fu, D. Priante, J.R.D. Retamal, C. Zhao, M. Ebaid, T.K. Ng, I. Ajia, S. Mitra, I.S. Roqan, B.S. Ooi, J.H. He, Nano Lett., 17 (2017) 1520-1528.

[8] D.F. Wang, A. Pierre, M.G. Kibria, K. Cui, X.G. Han, K.H. Bevan, H. Guo, S. Paradis, A.R. Hakima, Z.T. Mi, Nano Lett., 11 (2011) 2353-2357.

[9] M. Junaid, Y.T. Chen, J. Palisaitis, M. Garbrecht, C.L. Hsiao, P.O.Å. Persson, L. Hultman, J. Birch, Mater. Sci. Semicond. Process, 39 (2015) 702-710.

[10] M. Ebaid, J.H. Kang, S.W. Ryu, Semicond. Sci. Technol., 32 (2017) 013001.

[11] D. van Treeck, G. Calabrese, J.J.W. Goertz, V.M. Kaganer, O. Brandt, S. Fernandez-Garrido, L. Geelhaar, Nano Res., 11 (2018) 565-576.

[12] M. Wolz, C. Hauswald, T. Flissikowski, T. Gotschke, S. Fernandez-Garrido, O. Brandt, H.T.

Grahn, L. Geelhaar, H. Riechert, Nano Lett., 15 (2015) 3743-3747.

[13] G. Calabrese, P. Corfdir, G.H. Gao, C. Pfuller, A. Trampert, O. Brandt, L. Geelhaar, S.

Fernandez-Garrido, Appl. Phys. Lett., 108 (2016) 202101.

[14] G. Calabrese, S.V. Pettersen, C. Pfuller, M. Ramsteiner, J.K. Grepstad, O. Brandt, L. Geelhaar, S. Fernandez-Garrido, Nanotechnology, 28 (2017) 425602.

[15] B.J. May, A. Sarwar, R.C. Myers, Appl. Phys. Lett., 108 (2016) 141103.

[16] C. Ramesh, P. Tyagi, G. Gupta, M.S. Kumar, S.S. Kushvaha, Jpn. J. Appl. Phys., 58 (2019) 23-

28.

[17] C. Ramesh, P. Tyagi, G. Abhiram, G. Gupta, M. Senthil Kumar, S.S. Kushvaha, J. Cryst. Growth, 509 (2019) 23-28. 
[18] C. Ramesh, P. Tyagi, J. Kaswan, B.S. Yadav, A.K. Shukla, M. Senthil Kumar, S.S. Kushvaha, RSC Adv., 10 (2020) 2113-2122.

[19] S. Ruvimov, Z. Liliental-Weber, J. Washburn, K.J. Duxstad, E.E. Haller, Z.-F. Fan, S.N. Mohammad, W. Kim, A.E. Botchkarev, H. Morkoç, Appl. Phys. Lett., 69 (1996) 1556-1558. [20] B.P. Luther, S.E. Mohney, T.N. Jackson, Semicond. Sci. Technol., 13 (1998) 1322-1327. [21] A. Bengoechea-Encabo, F. Barbagini, S. Fernandez-Garrido, J. Grandal, J. Ristic, M.A. SanchezGarcia, E. Calleja, U. Jahn, E. Luna, A. Trampert, J. Cryst. Growth, 325 (2011) 89-92. [22] M. Sobanska, A. Wierzbicka, K. Klosek, J. Borysiuk, G. Tchutchulashvili, S. Gieraltowska, Z.R. Zytkiewicz, J. Cryst. Growth, 401 (2014) 657-660.

[23] M. Musolino, A. Tahraoui, S. Fernández-Garrido, O. Brandt, A. Trampert, L. Geelhaar, H. Riechert, Nanotechnology, 26 (2015) 085605.

[24] C.H. Chen, C. E. Cheng, C.C. Hsu, M.N. Chang, H.W. Shiu, F.S.S. Chien, J. Phys. D Appl. Phys., 45 (2012) 215307.

[25] G. Calabrese, G. Gao, D. van Treeck, P. Corfdir, C. Sinito, T. Auzelle, A. Trampert, L. Geelhaar, O. Brandt, S. Fernandez-Garrido, Nanotechnology, 30 (2019).

[26] C. Zhao, T.K. Ng, N.N. Wei, A. Prabaswara, M.S. Alias, B. Janjua, C. Shen, B.S. Ooi, Nano Lett., 16 (2016) 1056-1063.

[27] J. Ristic, E. Calleja, S. Fernandez-Garrido, L. Cerutti, A. Trampert, U. Jahn, K.H. Ploog, J. Cryst. Growth, 310 (2008) 4035-4045.

[28] V. Consonni, M. Knelangen, A. Trampert, L. Geelhaar, H. Riechert, Appl. Phys. Lett., 98 (2011) 071913.

[29] O. Brandt, S. Fernández-Garrido, J.K. Zettler, E. Luna, U. Jahn, C. Chèze, V.M. Kaganer, Cryst. Growth Des., 14 (2014) 2246-2253.

[30] K.A. Grossklaus, A. Banerjee, S. Jahangir, P. Bhattacharya, J.M. Millunchick, J. Cryst. Growth, 371 (2013) 142-147.

[31] L.E. Davis, N.C. MacDonald, P.W. Palmberg, G.E. Riach, R.E. Weber, Handbook of Auger Electron Spectroscopy, Second ed., Physical Electronics Division Perkin·Elmer Corporation, Minnesota, USA, 1976. 


\section{Table 1.}

Growth parameters and properties of GaN nanowire systems on Ti-film/n-Si(100)

\begin{tabular}{|c|c|c|c|c|c|c|}
\hline \multirow{2}{*}{\multicolumn{2}{|c|}{$\begin{array}{l}\text { Substrate and } \mathrm{Ga} \\
\text { cell temperatures } \\
\text { Fig. } \mathrm{T}_{\text {sub }} / \mathrm{T}_{\mathrm{Ga}}\left({ }^{\circ} \mathrm{C}\right)\end{array}$}} & \multicolumn{5}{|c|}{ GaN nanowires on Ti film } \\
\hline & & $\begin{array}{l}\text { Length (nm) } \\
\text { Growth time (h) }\end{array}$ & $\begin{array}{c}\text { Diameter } \\
(\mathrm{nm})\end{array}$ & $\begin{array}{l}{ }^{\mathrm{a}} \text { Surface } \\
\text { coverage }(\%)\end{array}$ & $\begin{array}{l}\text { Ga:N } \\
\text { ratio }\end{array}$ & Shape \\
\hline (1) & $680 / 865$ & $280-320(5)$ & $40-70$ & 45 & $1.0: 1.0$ & Irre. hexagonal \\
\hline \multirow[t]{2}{*}{ (2) } & $680 / 860$ & $150-210(4)-\mathrm{N}$ & $50-70$ & 40 & $1.0: 1.4$ & Irre. hexagonal \\
\hline & & $230-270(4)$ & $60-100$ & 56 & $1.0: 1.0$ & Irre. hexagonal \\
\hline (3) & $700 / 860$ & $200-240(4)$ & $40-70$ & 55 & $1.0: 1.2$ & Irre. hexagonal \\
\hline
\end{tabular}

\section{Table 2.}

Growth parameters and properties of GaN nanowire systems on Ti-foil

\begin{tabular}{|c|c|c|c|c|c|c|}
\hline \multirow{2}{*}{\multicolumn{2}{|c|}{$\begin{array}{l}\text { Substrate and } \mathrm{Ga} \\
\text { cell temperatures } \\
\text { Fig. } \mathrm{T}_{\mathrm{sub}} / \mathrm{T}_{\mathrm{Ga}}\left({ }^{\circ} \mathrm{C}\right)\end{array}$}} & \multicolumn{5}{|c|}{ GaN nanowires on Ti foil } \\
\hline & & $\begin{array}{l}\text { Length (nm) } \\
\text { Growth time (h) }\end{array}$ & $\begin{array}{c}\text { Diameter } \\
(\mathrm{nm})\end{array}$ & $\begin{array}{c}{ }^{\mathrm{a}} \text { Surface } \\
\text { coverage }(\%)\end{array}$ & $\begin{array}{l}\text { Ga:N } \\
\text { ratio }\end{array}$ & Shape \\
\hline \multirow[t]{2}{*}{$(5)(4)$} & $700 / 865$ & $220-305(4)-\mathrm{N}$ & $30-60$ & 47 & $1.0: 1.9$ & Some hexagonal \\
\hline & & $40-110(3)$ & $25-50$ & 27 & $1.0: 2.2$ & Irregular \\
\hline (6) & $680 / 835$ & $120-200(4)$ & $60-100$ & 40 & $1.0: 2.5$ & Some hexagonal \\
\hline (7) & $700 / 875$ & $220-380(4)-\mathrm{N}$ & $30-60$ & 40 & - & Irregular \\
\hline (9) & $700 / 825$ & $170-220(2.5)-\mathrm{N}$ & $\approx 50$ & 23 & $1.0: 3.7$ & Hexagonal \\
\hline
\end{tabular}

${ }^{a}$ Surface coverage: Percentage of the substrate surface covered by nanowires. Top surface areas of nanowires obtained using the ImgeJ software was used in the calculation of the coverage percentage. $\mathrm{N}$-With nitridation; Irre. - Irregular; Some hexagonal - Some nanowires are hexagonal and others are irregular hexagonal. 


\section{Figure Captions}

Fig. 1. SEM micrographs of (A) plan view and (B) $40^{\circ}$-tilt view of a $\mathrm{GaN}$ nanowire system grown on $300 \mathrm{~nm}$ Ti-film/n-Si(100) at the following conditions. $\mathrm{T}_{\mathrm{sub}}=680^{\circ} \mathrm{C} ; \mathrm{T}_{\mathrm{Ga}}$ cell $=865^{\circ} \mathrm{C}$; pre-nitridation at $650{ }^{\circ} \mathrm{C}$ for one hour; growth time $=5$ hours. (C) Auger electron spectrum of the GaN nanowires of $\mathrm{A}$.

Fig. 2. SEM micrographs of GaN nanowire systems grown on $300 \mathrm{~nm}$ Ti-film/n-Si(100) (A) with prenitridation and (C) without pre-nitridation at the following conditions: $\mathrm{T}_{\text {sub }}=680{ }^{\circ} \mathrm{C} ; \mathrm{T}_{\mathrm{Ga}}$ cell $=860{ }^{\circ} \mathrm{C}$; growth time $=4$ hours. The corresponding Auger electron spectra are shown at right sides.

Fig. 3. SEM micrographs of (A) plan view and (B) $40^{\circ}$-tilt view of a $\mathrm{GaN}$ nanowire system grown on $300 \mathrm{~nm}-\mathrm{Ti}$ film/ $\mathrm{Si}(100)$ at the following conditions: $\mathrm{T}_{\text {sub }}=700{ }^{\circ} \mathrm{C}$; without pre-nitridation; $\mathrm{T}_{\mathrm{Ga}}$ cell $=$ $860{ }^{\circ} \mathrm{C}$; growth time $=4$ hours. $(\mathrm{C})$ The corresponding Auger electron spectrum of the GaN nanowires.

Fig. 4. SEM micrographs of (A) plan view and (B) $40^{\circ}$-tilt view of a GaN nanowire system grown on Ti-foil at the following conditions: $\mathrm{T}_{\text {sub }}=700{ }^{\circ} \mathrm{C}$; without pre-nitridation; $\mathrm{T}_{\mathrm{Ga}}$ cell $=865^{\circ} \mathrm{C}$; growth time $=3$ hours. $(\mathrm{C})$ The corresponding Auger electron spectrum of the GaN nanowires.

Fig. 5. SEM micrographs of (A) plan view and (B) $40^{\circ}$-tilt view of a $\mathrm{GaN}$ nanowire system grown on Ti-foil at the following conditions: $\mathrm{T}_{\text {sub }}=700{ }^{\circ} \mathrm{C}$; pre-nitridation at $700{ }^{\circ} \mathrm{C}$ for one hour; $\mathrm{TGa}_{\text {cell }}=865$ ${ }^{\circ} \mathrm{C}$; growth time $=4$ hours. (C) The corresponding Auger electron spectrum of the GaN nanowires.

Fig. 6. (A) The $40^{\circ}$-tilt view of an SEM micrograph of a GaN nanowire system grown on Ti-foil at the following conditions: $\mathrm{T}_{\text {sub }}=680{ }^{\circ} \mathrm{C}$; after pre-nitridation; $\mathrm{T}_{\mathrm{Ga}}$ cell $=835^{\circ} \mathrm{C}$; growth time $=4$ hours $(\mathrm{B})$ Auger electron spectra of the GaN nanowire systems shown in A (black) and Fig. 5C (red).

Fig. 7. SEM micrographs of (A) plan view and (B) $40^{\circ}$-tilt view of a $\mathrm{GaN}$ nanowire system grown on Ti-foil at the following conditions: $\mathrm{T}_{\mathrm{sub}}=70{ }^{\circ} \mathrm{C}$; after pre-nitridation; $\mathrm{T}_{\mathrm{Ga}}$ cell $=875^{\circ} \mathrm{C}$; growth time $=$ 4 hours.

Fig. 8. SEM micrographs of (A) and (B) $40^{\circ}$-tilt view and (C) plan view of a GaN nanowire system grown on Ti-foil at the following conditions: (A) $\mathrm{T}_{\text {sub }}=700{ }^{\circ} \mathrm{C}$; no pre-nitridation; $\mathrm{T}_{\mathrm{Ga}}$ cell $=825{ }^{\circ} \mathrm{C}$; growth time $=180$ minutes. 
Fig. 9. SEM micrographs of (A) $40^{\circ}$-tilt view and (B) plan view of a GaN nanowire system grown on Ti-foil at the following conditions; $\mathrm{T}_{\text {sub }}=700{ }^{\circ} \mathrm{C}$; after pre-nitridation; $\mathrm{T}_{\mathrm{Ga}}$ cell $=825^{\circ} \mathrm{C}$; growth time $=2.5$ hours $(\mathrm{C})$ The Auger electron spectrum of the GaN nanowire system shown in $\mathrm{A}$.

Fig. 10. SEM micrographs of (A) and (C) plan view and (B) $40^{\circ}$-tilt view of a GaN nanowire system grown on Ti-foil at the following conditions: $\mathrm{T}_{\text {sub }}=690{ }^{\circ} \mathrm{C}$; after pre-nitridation; $\mathrm{T}_{\mathrm{Ga}}$ cell $=865{ }^{\circ} \mathrm{C}$; deposition time $=4$ hours. (B) The corresponding Auger electron spectrum of the GaN nanowires. 


\section{Figures}
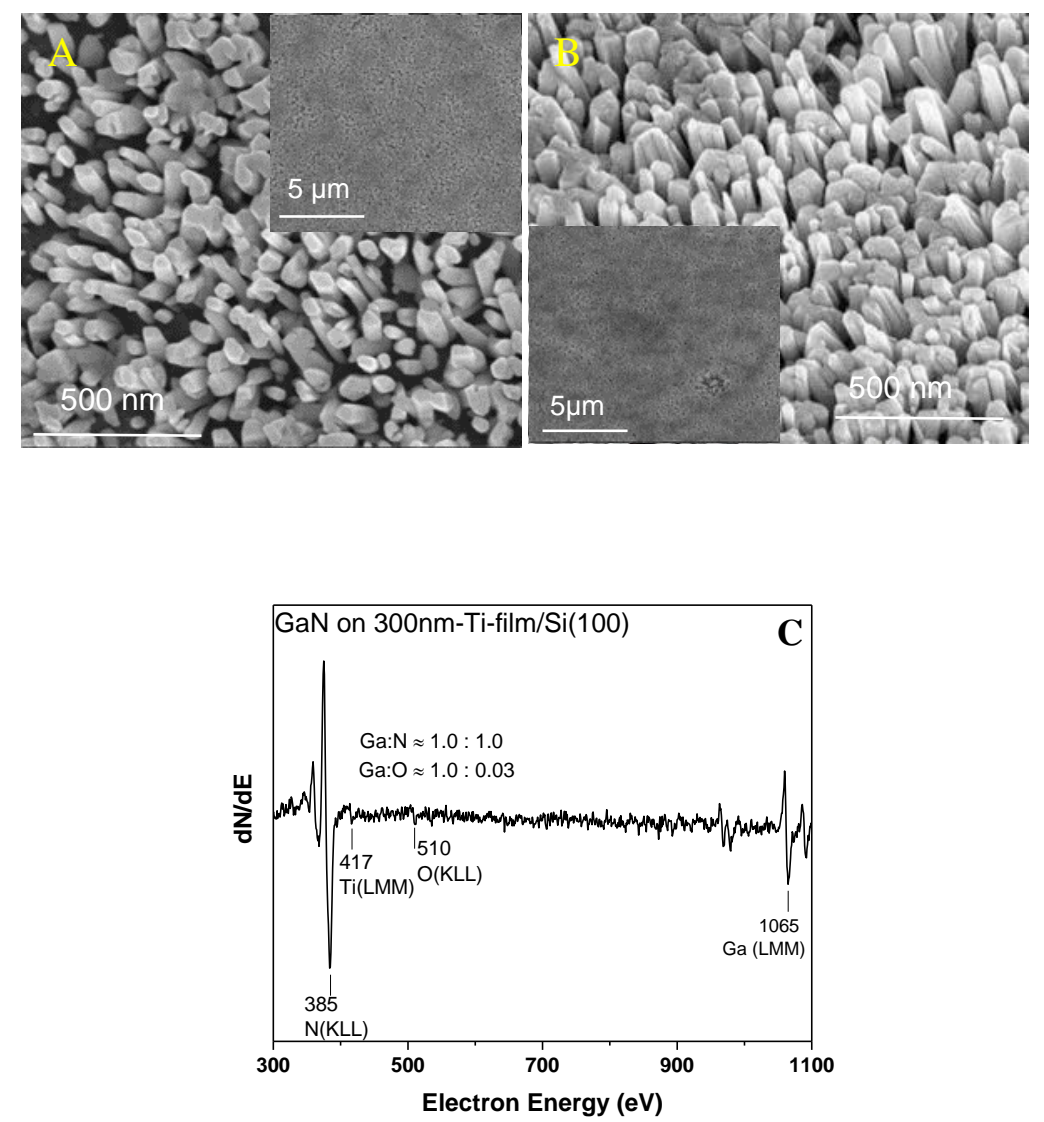

Fig. 1.

SEM micrographs of (A) plan view and (B) $40^{\circ}$-tilt view of a GaN nanowire system grown on $300 \mathrm{~nm}$ Ti-film/n-Si(100) at the following conditions. $\mathrm{T}_{\text {sub }}=680{ }^{\circ} \mathrm{C} ; \mathrm{T}_{\mathrm{Ga}}$ cell $=865^{\circ} \mathrm{C}$; pre-nitridation at $650{ }^{\circ} \mathrm{C}$ for one hour; growth time $=5$ hours. (C) Auger electron spectrum of the GaN nanowires of A; AES Ti to $\mathrm{N}=1.0: 21.5$. AES lines intensity was corrected using the relative sensitivity factors at $3 \mathrm{keV}$ beam energy [31]. 

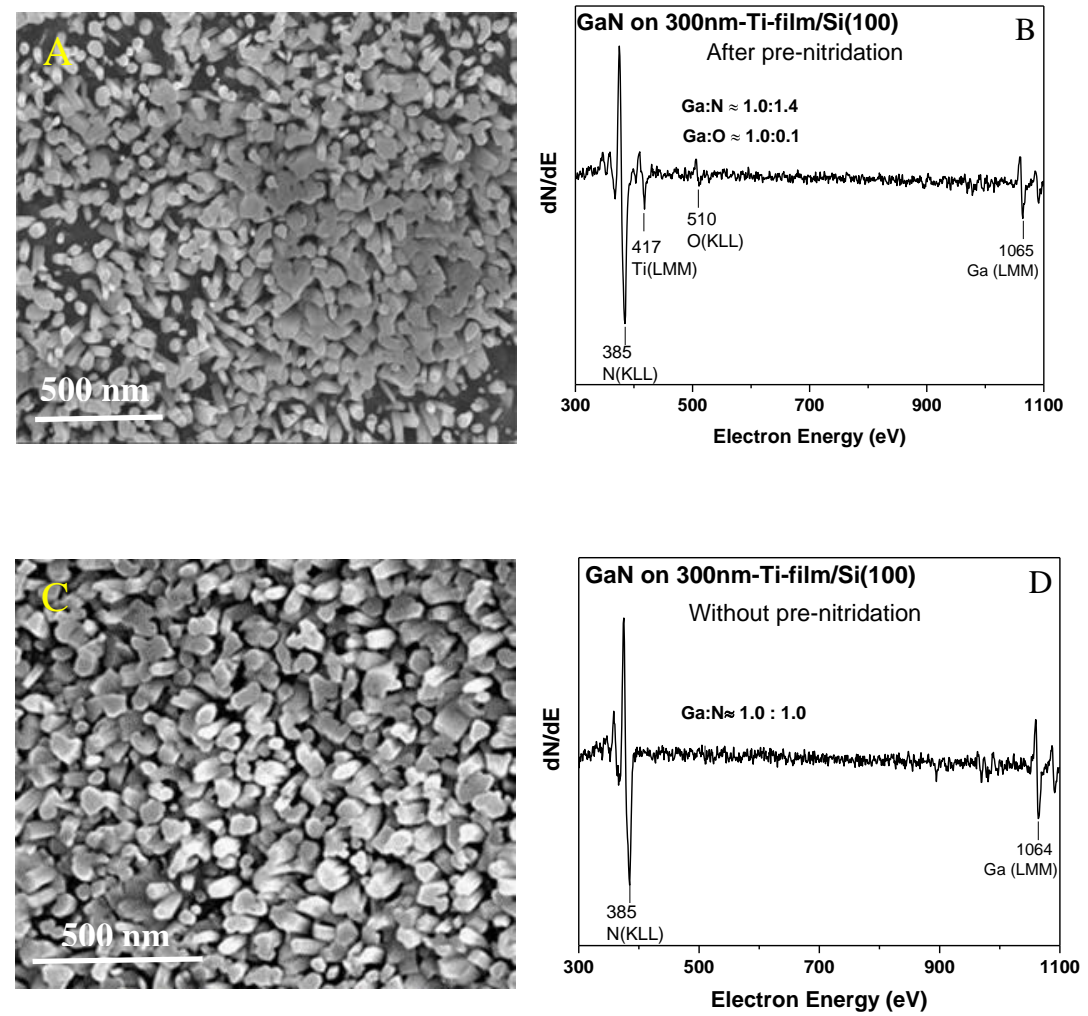

Fig. 2.

SEM micrographs of GaN nanowire systems grown on $300 \mathrm{~nm}$ Ti-film $/ \mathrm{n}-\mathrm{Si}(100)$ (A) with prenitridation; $\mathrm{AES}$ Ti to $\mathrm{N}=1.0: 6.0$, and (C) without pre-nitridation at following conditions: $\mathrm{T}_{\text {sub }}=680$ ${ }^{\circ} \mathrm{C} ; \mathrm{T}_{\mathrm{Ga}}$ cell $=860{ }^{\circ} \mathrm{C}$; growth time $=4$ hours. The corresponding Auger electron spectra are shown in $\mathrm{B}$ and $\mathrm{D}$. 

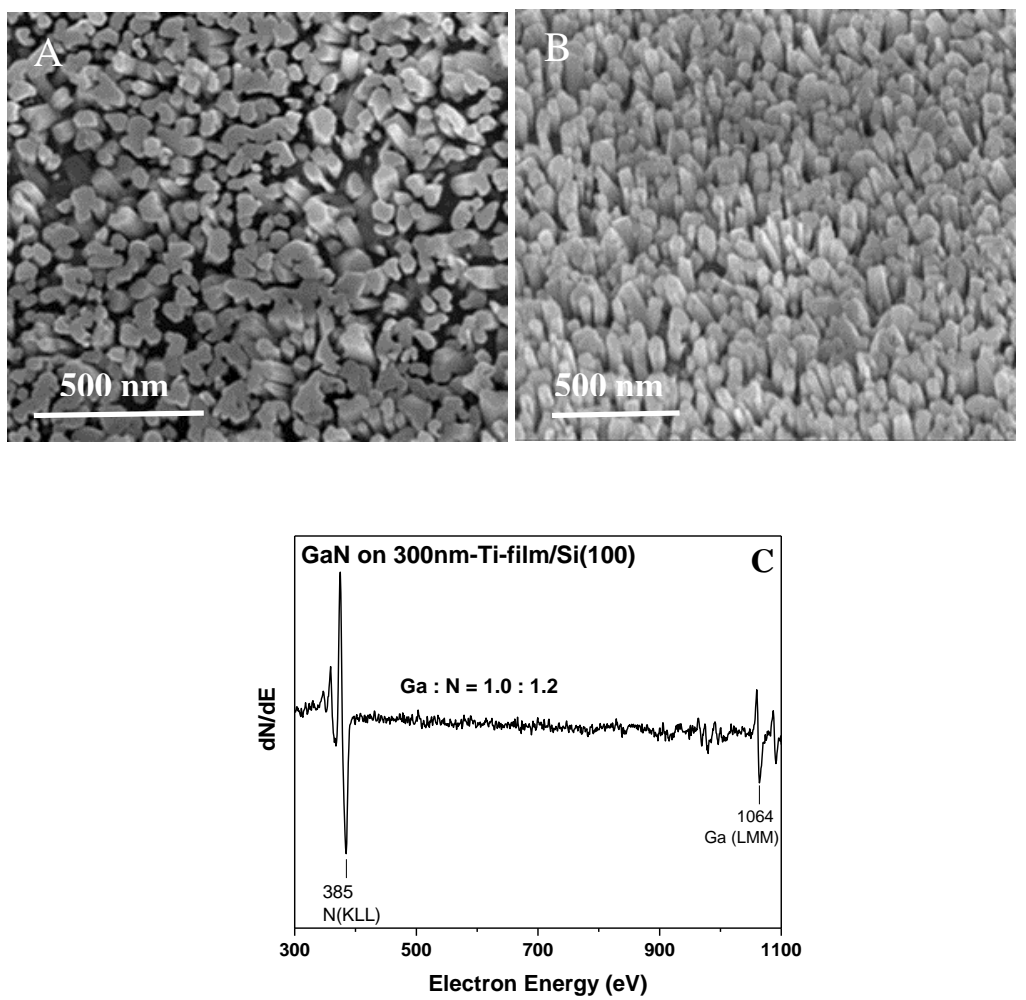

Fig. 3.

SEM micrographs of (A) plan view and (B) $40^{\circ}$-tilt view of a GaN nanowire system grown on $300 \mathrm{~nm}$ $\mathrm{Ti}$ film/ $\mathrm{Si}(100)$ at the following conditions: $\mathrm{T}_{\text {sub }}=700{ }^{\circ} \mathrm{C}$; without pre-nitridation; $\mathrm{T}_{\mathrm{Ga}}$ cell $=860{ }^{\circ} \mathrm{C}$; growth time $=4$ hours. $(\mathrm{C})$ The corresponding Auger electron spectrum of the GaN nanowires. 

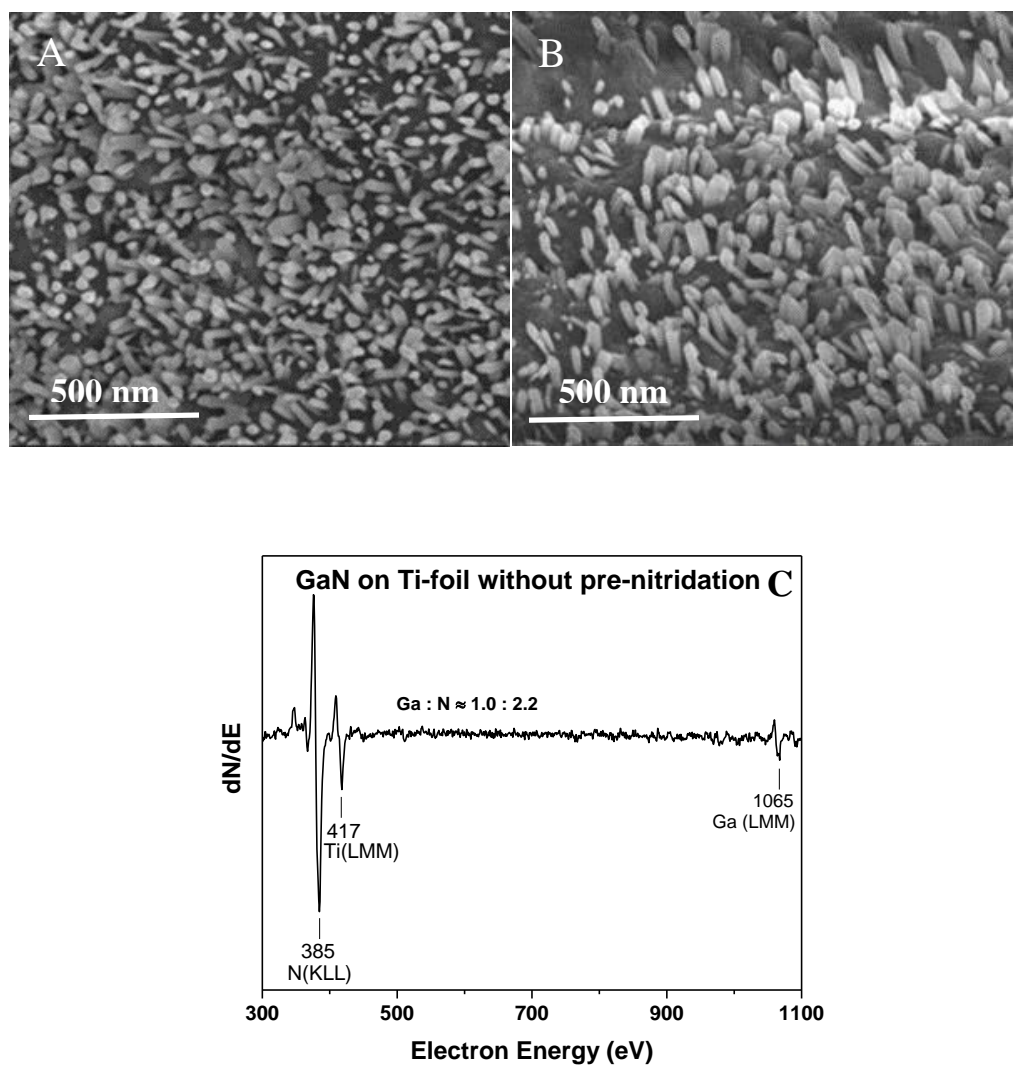

Fig. 4.

SEM micrographs of (A) plan view and (B) $40^{\circ}$-tilt view of a $\mathrm{GaN}$ nanowire system grown on Ti-foil at the following conditions: $\mathrm{T}_{\text {sub }}=700{ }^{\circ} \mathrm{C}$; without pre-nitridation; $\mathrm{T}_{\mathrm{Ga}}$ cell $=865^{\circ} \mathrm{C}$; growth time $=3$ hours. (C) The corresponding Auger electron spectrum of the GaN nanowires; AES Ti to $\mathrm{N}=1.0: 4.3$ 

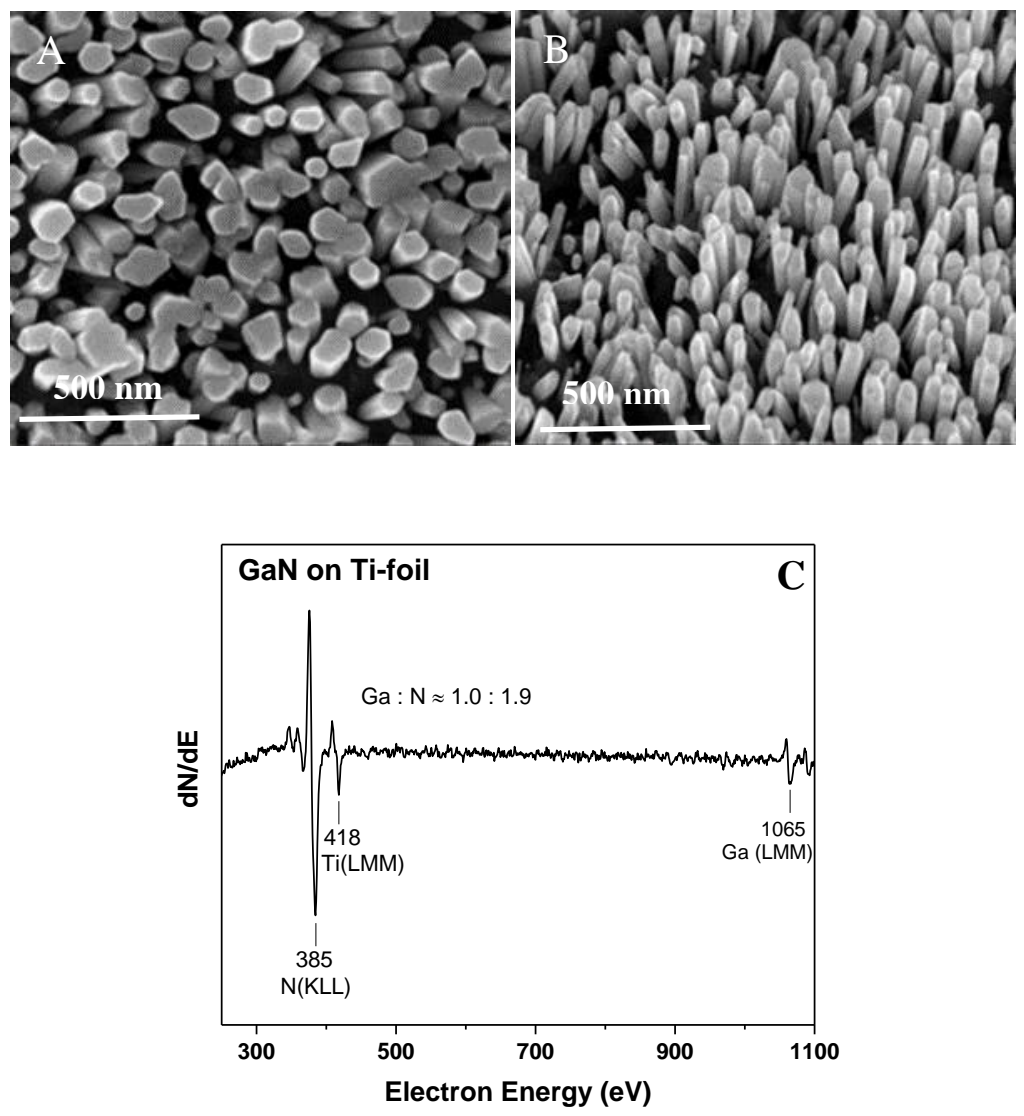

Fig. 5.

SEM micrographs of (A) plan view and (B) $40^{\circ}$-tilt view of a GaN nanowire system grown on Ti-foil at the following conditions: $\mathrm{T}_{\mathrm{sub}}=700{ }^{\circ} \mathrm{C}$; pre-nitridation at $700{ }^{\circ} \mathrm{C}$ for one hour; $\mathrm{T}_{\mathrm{Ga}}$ cell $=865^{\circ} \mathrm{C}$; growth time $=4$ hours. (C) The corresponding Auger electron spectrum of the GaN nanowires; AES Ti to $\mathrm{N}=1.0: 5.1$ 

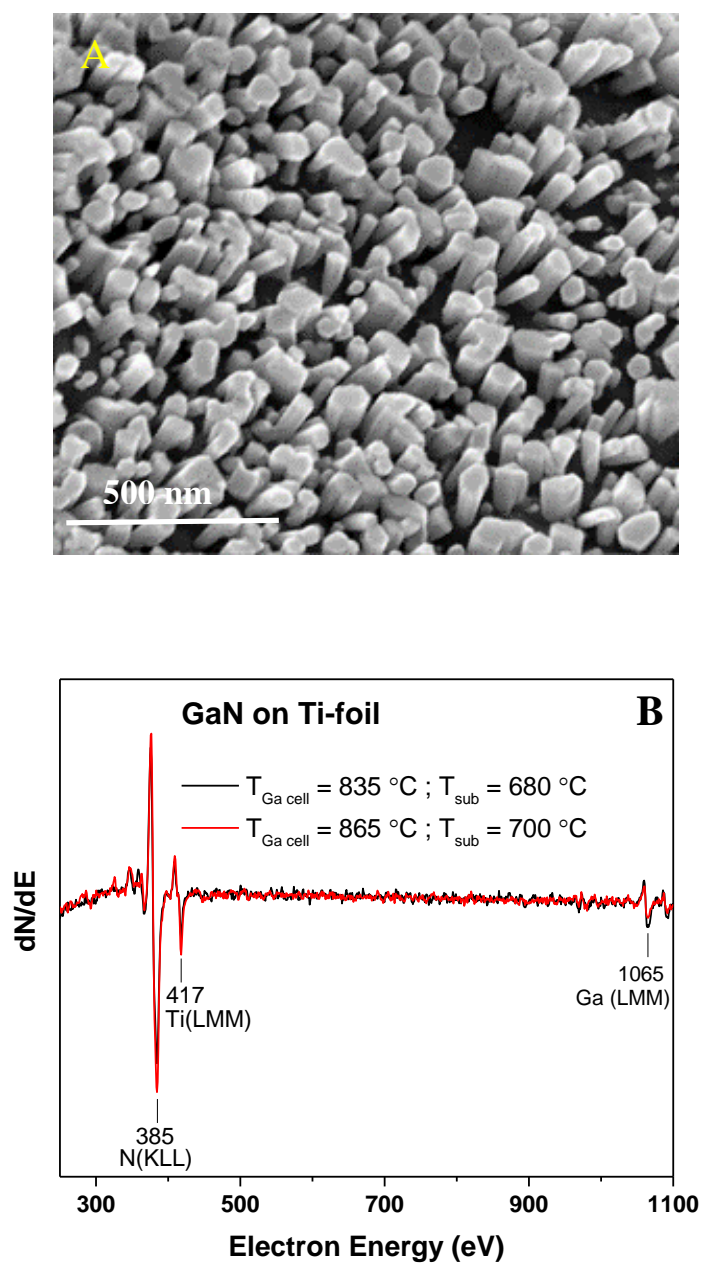

Fig. 6.

(A) The $40^{\circ}$-tilt view of an SEM micrograph of a GaN nanowire system grown on Ti-foil at the following conditions: $\mathrm{T}_{\mathrm{sub}}=680^{\circ} \mathrm{C}$; after pre-nitridation; $\mathrm{T}_{\mathrm{Ga}}$ cell $=835^{\circ} \mathrm{C}$; growth time $=4$ hours $(\mathrm{B})$ Auger electron spectra of the GaN nanowire systems shown in A (black) and from Fig. 5C (red); AES Ti to $\mathrm{N}=1.0: 5.2$ (black spectrum) 

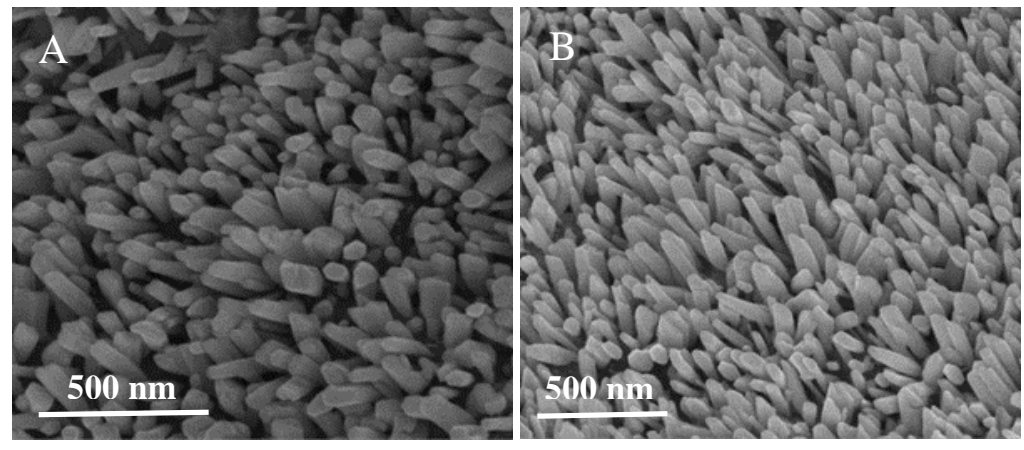

Fig. 7.

SEM micrographs of (A) plan view and (B) $40^{\circ}$-tilt view of a $\mathrm{GaN}$ nanowire system grown on Ti-foil at the following conditions: $\mathrm{T}_{\text {sub }}=700{ }^{\circ} \mathrm{C}$; after pre-nitridation; $\mathrm{T}_{\mathrm{Ga}}$ cell $=875^{\circ} \mathrm{C}$; growth time $=4$ hours. 


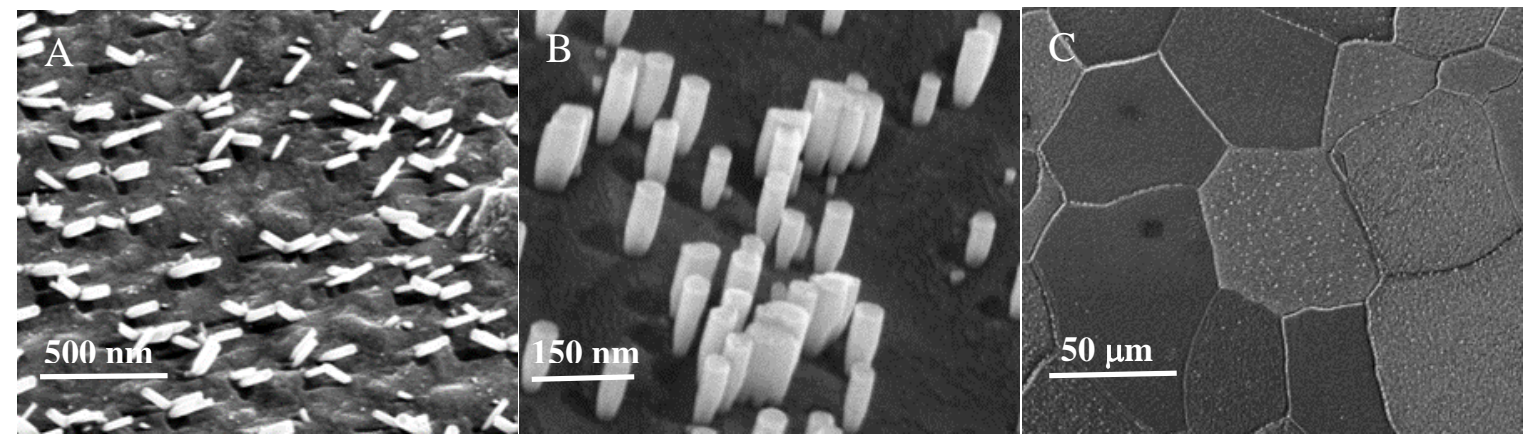

Fig. 8.

SEM micrographs of (A) and (B) $40^{\circ}$-tilt view and (C) plan view of a GaN nanowire system grown on Ti-foil at the following conditions: (A) $\mathrm{T}_{\text {sub }}=700{ }^{\circ} \mathrm{C}$; no pre-nitridation; $\mathrm{T}_{\mathrm{Ga}}$ cell $=825{ }^{\circ} \mathrm{C}$; growth time $=180$ minutes. 

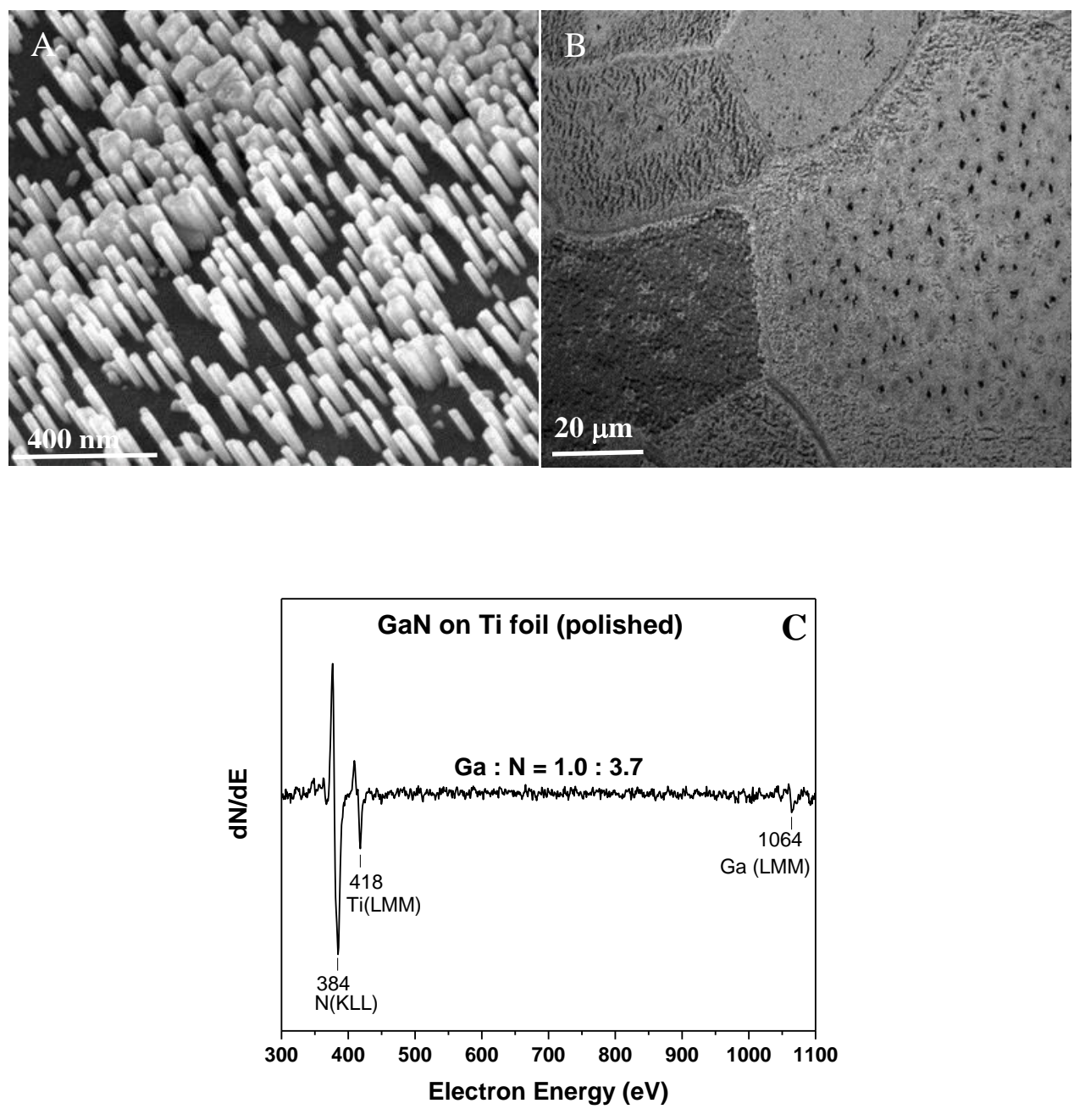

Fig. 9.

SEM micrographs of (A) $40^{\circ}$-tilt view and (B) plan view of a GaN nanowire system grown on Ti-foil at the following conditions; $\mathrm{T}_{\text {sub }}=700{ }^{\circ} \mathrm{C}$; after pre-nitridation; $\mathrm{T}_{\mathrm{Ga}}$ cell $=825{ }^{\circ} \mathrm{C}$; growth time $=2.5$ hours $(\mathrm{C})$ The Auger electron spectrum of the GaN nanowire system shown in A; AES Ti to N = 1.0 : 4.6. 

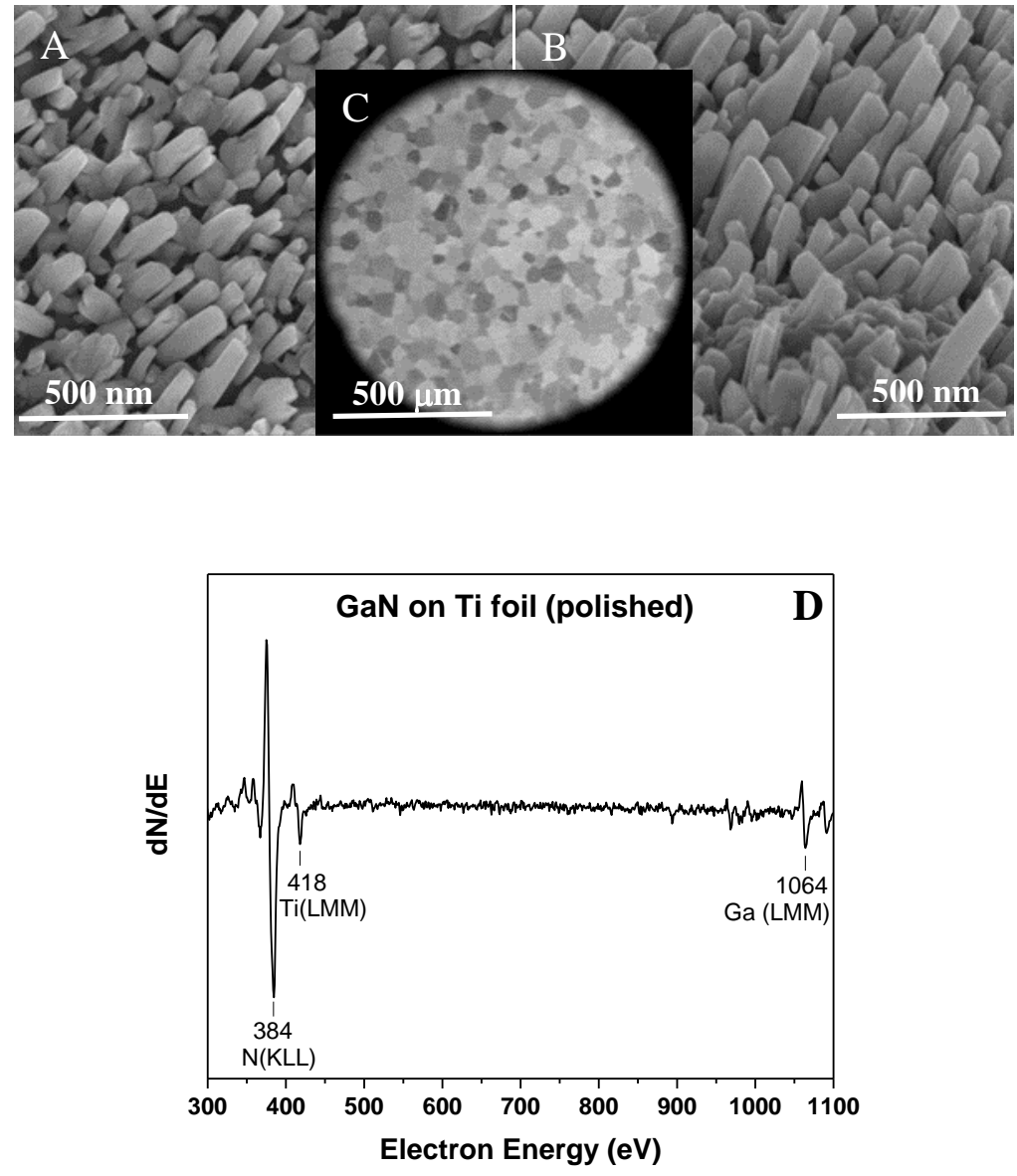

Fig. 10.

SEM micrographs of (A) and (C) plan view and (B) $40^{\circ}$-tilt view of a GaN nanowire system grown on Ti-foil at the following conditions: $\mathrm{T}_{\mathrm{sub}}=690^{\circ} \mathrm{C}$; after pre-nitridation; $\mathrm{T}_{\mathrm{Ga}}$ cell $=865^{\circ} \mathrm{C}$; deposition time $=4$ hours. (D) The corresponding Auger electron spectrum of the GaN nanowires; AES Ti to $\mathrm{N}=$ $1.0: 7.4$ 


\section{Supplementary Information}
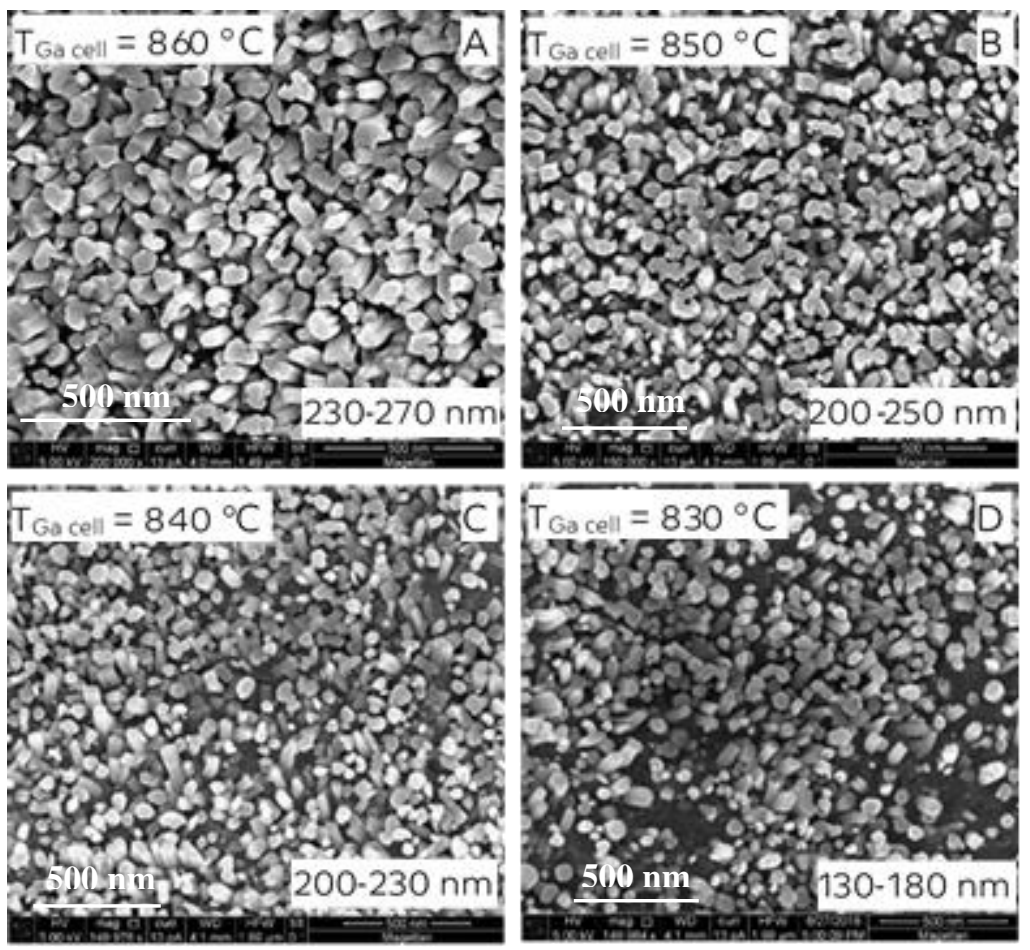

Fig. S1. SEM micrographs of GaN nanowire systems grown on $300 \mathrm{~nm}-\mathrm{Ti}$ film/Si(100) at different Ga cell temperatures under the following conditions: $\mathrm{T}_{\mathrm{sub}}=680{ }^{\circ} \mathrm{C}$; without pre-nitridation; growth time $=$ 4 hours. The lengths of nanowires are indicated in the insets. 